Research Paper

\title{
Mucosal Delivery of ACNPV Baculovirus Driving Expression of the Gal-Lectin LC3 Fragment Confers Protection against Amoebic Liver Abscess in Hamster
}

\author{
DM Meneses-Ruiz1, JP Laclette 1, H Aguilar-Díaz¹, J Hernández-Ruiz³, A Luz-Madrigal2, A Sampieri², L Vaca² \\ and JC Carrero ${ }^{1 \times}$
}

1. Department of Immunology, Instituto de Investigaciones Biomédicas, Universidad Nacional Autónoma de México. A.P. 70228, México D.F., México.

2. Department of Cell Biology, Instituto de Fisiología Celular, Universidad Nacional Autónoma de México. A.P. 70228, México D.F., México.

3. Unit of Experimental Medicine, Facultad de Medicina. Universidad Nacional Autónoma de México. A.P. 70228, México D.F., México.

$\triangle$ Corresponding author: Tel.: + 52 5556229220; fax: +52 5556223369. E-mail address: carrero@servidor.unam.mx (J.C. Carrero)

(C) Ivyspring International Publisher. This is an open-access article distributed under the terms of the Creative Commons License (http://creativecommons.org/ licenses/by-nc-nd/3.0/). Reproduction is permitted for personal, noncommercial use, provided that the article is in whole, unmodified, and properly cited.

Received: 2011.09.01; Accepted: 2011.10.01; Published: 2011.11.01

\begin{abstract}
Mucosal vaccination against amoebiasis using the Gal-lectin of E. histolytica has been proposed as one of the leading strategies for controlling this human disease. However, most mucosal adjuvants used are toxic and the identification of safe delivery systems is necessary. Here, we evaluate the potential of a recombinant Autographa californica baculovirus driving the expression of the LC3 fragment of the Gal-lectin to confer protection against amoebic liver abscess (ALA) in hamsters following oral or nasal immunization. Hamsters immunized by oral route showed complete absence $(57.9 \%)$ or partial development $(21 \%)$ of ALA, resulting in some protection in $78.9 \%$ of animals when compared with the wild type baculovirus and sham control groups. In contrast, nasal immunization conferred only $21 \%$ of protection efficacy. Levels of ALA protection showed lineal correlation with the development of an anti-amoebic cellular immune response evaluated in spleens, but not with the induction of seric lgG anti-amoeba antibodies. These results suggest that baculovirus driving the expression of $E$. histolytica vaccine candidate antigens is useful for inducing protective cellular and humoral immune responses following oral immunization, and therefore it could be used as a system for mucosal delivery of an anti-amoebic vaccine.
\end{abstract}

Key words: Amoeba, LC3 Gal-lectin, Baculovirus, ALA, protection, hamster

\section{Introduction}

Entamoeba histolytica is a protozoan parasite which causes dysentery and amoebic liver abscess (ALA) disease in humans. It is responsible for 40, 000 to 100,000 deaths annually, ranking it as the second highest cause of mortality attributed to a protozoan parasitic disease (1). In Mexico, amoebiasis is ranked as the sixth highest cause of morbidity (2), with the last national seroepidemiological survey showing a seroprevalence rate of $8.4 \%$ in general population using an indirect hemagglutination test (3). Amoebiasis is most frequent in developing countries where environmental and population hygiene measures can be deficient. Although pharmacological treatment with metronidazole is effective, the still widespread prevalence of the disease as well as the side effects associated with the drug positions vaccination against amoebiasis an attractive alternative (4). Ideally, an anti-amoebic vaccine should confer protection against 
mucosal colonization by the trophozoites, avoiding intestinal amoebic dysentery, and consequently, the extraintestinal ALA disease (5).

Several candidates for amoebic vaccination have been proposed by different groups with a galactose/N-acetyl-D-galactosamine-inhibitable lectin (Gal-lectin) identified as the leading candidate due to its pivotal role in parasite virulence and adhesion to the intestinal mucosa (6). Diverse studies have demonstrated the potential of native or fractionated Gal-lectin protein to confer protection against the development of ALA in gerbils using Freund's complete or incomplete adjuvants, Titermax adjuvant and/or CpG-oligonucleotides, (7-10). Similar results have been obtained using attenuated Yersinia and Salmonella vectors $(11,12)$ and even, by passive immunization with anti-Gal lectin IgG antibodies (13). A fragment of the Gal-lectin heavy subunit named LC3 $(52 \mathrm{kDa})$, which includes the carbohydrate-binding domain (8), has been shown to be one of the main targets for IgG and IgA anti-Gal lectin antibodies recovered from patient's serum and feces, respectively (14-16). Using Balb/c mice and non-human primates, immunization with cholera toxin-adjuvanted LC3 by oral or nasal routes, resulted in elevated serum IgG and fecal IgA anti-native Gal-lectin antibody levels with the capacity to inhibit E. histolytica trophozoites galactose-specific adherence to $\mathrm{CHO}$ cells in vitro (17, 18). In another study, LC3 fragment conferred protection in gerbils against ALA (71\%) following intraperitoneal immunization before intraportal challenge with virulent trophozoites (8).

In addition to inducing an effective local immune response, oral immunization has also been used to evaluate the ability of anti-E. histolytica vaccine candidates to induce protection again extraintestinal amoebiasis. Thus, recombinant complete Gal-lectin or its component fragments LC3 and LecA, the serine-rich protein SREHP, the $112 \mathrm{kDa}$ adhesin EhADH112 and a $30 \mathrm{kDa}$ protein belonging to the collagen-binding proteins familiy, have been shown to confer differing levels of protection against ALA development in hamsters and gerbils following oral immunization, suggesting a functional interaction between mucosal and systemic compartments (4, 7-13, 19-26). However, current limitations associated with oral or nasal immunization in humans include the lack of safe mucosal adjuvants. Most adjuvants used with animal models in immunization/protection trials are not acceptable for use in humans, including Freund's complete and incomplete adjuvant, cholera toxin (CT) and the attenuated bacterial and yeast stocks mentioned previously $(11,12,22,24)$. Thus, the search for safe adjuvants and optimum antigen de- livery strategies that are capable of inducing effective local protective immune responses against E. histolytica is a matter of fundamental interest. Diverse viral vectors have been studied to this end. One of the most promising is Autographa Californica, an insect enveloped double-stranded DNA nucleopolyhedrovirus (AcNPV) which can drive the expression of foreign genes in mammalian cells without causing cytotoxic effects (27).

AcNPV has been shown to possess a strong adjuvantig capacity in mammals, promoting enhanced humoral and cellular immune responses against co-administered antigens. In vitro, AcNPV induces maturation of antigen presenting cells (APCs) and production of proinflammatory cytokines and chemokines, via TLR9/MyD88-dependent pathways (28, 29), supporting its immunogenicity and potential as delivery vaccine vector. Numerous studies have demonstrated that recombinant baculovirus expressing target antigens on their surface (baculovirus display) and/or driving the cytomegalovirus (CMV) promoter-dependent expression (baculovirus expression) confer protection against a broad variety of pathogens. Effective immune protection has been reported in murine models using one or both systems for several viral and parasitic diseases (30-41).

In this work we demonstrate for the first time protection against the development of ALA in hamsters induced by oral immunization with an AcNPV baculovirus driving host cell expression of the E. histolytica LC3 Gal-lectin fragment. We show that oral immunization (in the absence of any type of adjuvant) using recombinant AcNPV baculovirus provide effective protection against ALA when compared to nasal immunization. These results position oral immunization with LC3-expressing baculovirus as a feasible tool for effective vaccination against hepatic amoebiasis.

\section{Materials and methods}

Cells and cultures. Axenic HM1: IMSS trophozoites were maintained in TYI-S-33 medium supplemented with $15 \%$ of adult bovine serum (Biofluids International Inc., MD, USA) and 3\% of Diamond's vitamin mix (JRH Biosciences, Kansas, USA) in anaerobic conditions and incubated at $37^{\circ} \mathrm{C}$. Trophozoites virulence was maintained trough successive passages into hamster's liver and recovering trophozoites from the induced ALA.

sf9 insect cells (Spodoptera frugiperda) (Invitrogen, San Diego, CA) were cultivated as monolayer or suspension in Grace's medium (Invitrogen, USA) supplemented with $10 \%$ heat-inactivated fetal bovine serum (Invitrogen, Carlsbad, CA), 1\% penicillin/ 
streptomycine $(100 \mathrm{U} / \mathrm{ml})$ and $0,1 \%$ pluronic F-68 (Invitrogen, USA) and maintained in anaerobic conditions at $27^{\circ} \mathrm{C}$. Cultures in suspension were additionally maintained with constant shaking at $100 \mathrm{rpm}$. HepG2 cells from ATCC were cultivated in DMEM medium +L-glutamine (Invitrogen, USA) supplemented with FBS and 1\% penicillin/streptomycine and maintained at $37^{\circ} \mathrm{C}$ with $5 \% \mathrm{CO}_{2}$ and $95 \%$ humidity.

AcNPV-LC3 baculovirus production. The LC3 coding region (amino acid 773 to 1127) of the Gal-lectin heavy subunit (8) was amplified from $E$. histolytica HM1: IMSS DNA using the following primers: sense primer FLC3HCMV (5 ' TAGAAAGCTTATGTGTTCTAGTTTAACATGTCCA $\left.-3^{\prime}\right)$ and antisense primer RSLC3XCMV (5 ' TTTTTCTAGATTAACATGTTTTCTTTGTGTAAATA G-3'). The PCR product was cloned into Hind III/XbaI sites of plasmid pBlueCMV (42) to generate the construct $\mathrm{pBlueCMV-LC3}$. The LC3 product and the construct were checked for integrity of the ORF by sequencing in an automatic sequencer ABI-Prism 2.0. The recombinant baculovirus AcNPV-LC3 was generated by recombination of the CMV-LC3 cassette with AcNPV DNA following the manufacturer's instructions of the Bac-N-Blue Transfection Kit (Invitrogen, Carlsbad, CA). Recombinant AcNPV-LC3 was identified by reconstitution of the lac $Z$ gene and $\beta$-galactosidase expression determined by addition of 5-bromine-4-chlorine-3-indolil- $\beta$-D-galactoside (X-gal) $(150 \mu \mathrm{g} / \mathrm{ml})$ to infected sf9 cell supernatants. Viral DNA was isolated from positive supernatant following the manufacturer's instructions of the Bac-N-Blue Transfection Kit (Invitrogen, Carlsbad, CA). Presence of the LC3 fragment was confirmed by PCR amplification and its sequence confirmed by DNA sequencing.

Recombinant AcNPV-LC3 baculovirus was amplified by infecting sf9 insect cells at a multiplicity of infection (MOI) of 0.1 and purifying them from culture's supernatants 6 days after infection by centrifugation at $6,000 \mathrm{~g}$ for $15 \mathrm{~min}$ at $4^{\circ} \mathrm{C}$. Afterwards, baculovirus was concentrated by ultracentrifugation in a SW28 rotor (Beckman) at 141, $000 \mathrm{~g}$ and $4^{\circ} \mathrm{C}$ during 90 min, resuspended in PBS, loaded onto a $10-50 \%$ (wt/vol) sucrose gradient and centrifuged at same conditions. Finally, the virus-containing interphase layer was collected, diluted in PBS, centrifuged as before and the pelleted virus titered by using a plaque assay following the manufacturer's instructions (Invitrogen). Wild type virus (AcNPV-WT) was amplified and titered following the same protocol as for AcNPV-LC3.
HepG2 cells transduction and Western blotting. HepG2 cells $\left(1 \times 10^{5}\right)$ were transducted with 0.1 MOI of AcNPV-LC3 and analyzed for LC3 expression by Western blotting on cellular extracts obtained at 48 h post-transduction. Shortly, total extract $(50 \mu \mathrm{g} /$ well $)$ was run on denaturalized SDS-PAGE and the protein transferred to nitrocellulose membranes by electrophoresis. Blotted membranes were blocked with 3\% BSA during $2 \mathrm{~h}$ at room temperature and incubated with a rabbit polyclonal anti-Gal-lectin heavy subunit antiserum (kindly provided by Rosario López, Unidad de Medicina Experimental, Facultad de Medicina, UNAM) during $1 \mathrm{~h}$ at $37{ }^{\circ} \mathrm{C}$. After three washes with PBS-Tween 20, the membrane was incubated with an HRP-conjugated anti-rabbit IgG antibody (Zymed laboratories, San Francisco, CA. USA) during $1 \mathrm{~h}$ at $37^{\circ} \mathrm{C}$. Finally, the membrane was washed as before and the immune complexes revealed by chemiluminescence using ECL Plus Western Blotting Detection System kit (GE Healthcare). The antibodies were used to 1:1000 dilution.

Immunization and challenge protocols. Male Syrian golden hamsters (Mesocricetus auratus) 4 to 6 weeks of age were maintained free of pathogens with water and food ad libitum. Following a protocol approved by the Institutional Animal Care Committee, animals were divided in 6 groups of 19 hamsters each: two non-treated groups (non-infected and infected sham groups) and four immunized groups. Immunized groups comprised two by oral route with WT $(\mathrm{O}$ AcNPV-WT) or LC3 recombinant (O AcNPV-LC3) baculovirus and two groups by nasal route with WT (N AcNPV-WT) and LC3 recombinant ( $\mathrm{N}$ AcNPV-LC3) baculovirus. Light anesthetized hamsters were administered with three dosis at two weeks intervals with $1 \times 10^{8}$ PFU prepared in $200 \mu \mathrm{l} 0.2 \mathrm{M} \mathrm{Na}$ $\mathrm{HCO}_{3}, \mathrm{pH} 8.3$ for oral and in $20 \mu \mathrm{l}$ PBS, $\mathrm{pH} 7.4$ for nasal deliveries. Oral administration was done by using a neonatal feeding tube in previously Sevorane sedated animals, whereas nasal administration was done by releasing $10 \mu \mathrm{l}$ per each nostril using a pipette. Infected sham group received in the same animals $200 \mu \mathrm{l}$ of $\mathrm{NaHCO}_{3}$ and $20 \mu \mathrm{l}$ of PBS by oral and nasal route, respectively. Two weeks after the last immunization, all animal, except the non-infected sham group, were infected by intra-portal route with virulent E. histolytica trophozoites. In brief, animal were anesthetized with sodium pentobarbital (50 $\mathrm{mg} / \mathrm{kg}$; Anestesal, Pfizer) , a laparatomy practiced in aseptic conditions in order to expose the portal vein and $10^{6}$ trophozoites in $100 \mu \mathrm{l}$ PBS were directly injected (43). The site of injection was immediately obtruded by applying a gel foam pad, intestine carefully returned to the abdominal cavity, and the abdominal 
layers sutured with surgical staples (Reflex 9, USA). Blood samples were collected from all animals before treatment (day 0) and after immunization prior to challenge (day 35). Sera were obtained by centrifugation and stored at $-70{ }^{\circ} \mathrm{C}$ until use.

Liver histology. Seven days after the challenge, hamsters were sacrificed under anesthesia and the liver excised. General liver damage was recorded by macroscopic features, weight, distribution and size of abscesses. Coronal cuts from whole liver were fixed in $4 \%$ formaldehyde-PBS during $24 \mathrm{~h}$, whereas others fragments were included in Tissue Freezing Medium (Leica) and cut in $10 \mu$ for Hematoxylin-Eosin staining.

Antibodies determination by ELISA. Ninety six well plates were coated overnight with $500 \mathrm{ng} /$ well of trophozoite's total extract in carbonate buffer, $\mathrm{pH} 9.6$ at $4^{\circ} \mathrm{C}$. After blocking with $1 \%$ BSA-Tween 20 , hamster's sera diluted in 1\% BSA (1:50) were added to the wells and incubated $1 \mathrm{~h}$ at $37^{\circ} \mathrm{C}$. After extensive washings with PBS-Tween 20, a HRP-conjugated anti-hamster IgG antibody (Becton Dickinson, USA) was added to 1:1000 dilution and incubated for $1 \mathrm{~h}$ at $37^{\circ} \mathrm{C}$. Finally, the wells were washed and the antigen-antibody complexes were developed with OPD and read at $490 \mathrm{~nm}$ in a spectrophotometer.

Spleen cells proliferation by flow cytometry. After sacrifice at 7 days post-challenge, hamster's spleens were removed and its cellular fraction obtained by perfusion in supplemented RPMI medium. The white cells, mostly lymphocytes, were cultivated at $10^{5}$ cells/well in 96-well plates in RPMI medium alone or stimulated with concanavalin A $(1 \mu \mathrm{g} /$ well $)$, or amebic total extract (50 $\mu \mathrm{g} /$ well), at $37^{\circ} \mathrm{C}$ with $5 \%$ $\mathrm{CO}_{2}$ and $95 \%$ humidity for $72 \mathrm{~h}$. Finally, cells were harvested and fixed with formaldehyde at $3.7 \%$. All the treatments were carried out by triplicate for each animal. Samples were analyzed on a FACSCanto II flow cytometer (BD). A minimum of 20,000 events were collected and analyzed with the DIVA software from the instrument's manufacturer. Proliferation data from each experimental group was obtained by pooling the means of the triplicate for each animal and is presented as mean \pm SD.

Statistical analysis. The comparison of the infection rates between the immunized groups and their respective sham controls was done using Fisher's exact test. The Kruskal-Wallis test was used to compare antibody levels between groups. A P value $<0.05$ was considered statistically significant in these analyses. All statistical analyses were performed using SPSS statistical software version 19.0.

\section{Results}

Mammalian cells transduced with recombinant baculovirus AcNPV-LC3 efficiently expressed LC3 protein. The baculovirus AcNPV-LC3 was generated co-transfecting the transference plasmid pBlueCMV-LC3 (Fig. 1A) with linear DNA of AcNPV in sf9 insect cells. Purified recombinant viral particles were used to examine the capacity of recombinant baculovirus for driving the expression of LC3 in HepG2 mammalian cells. Western blot on $48 \mathrm{~h}$ transduced cells using an anti-Gal-lectin polyclonal antibody, showed recognition of a band with a molecular weight of approximately $50 \mathrm{kDa}$, which correspond to the expected weight for LC3 (Fig. 1B lane 3). WB on non-transduced HepG2 cells did not recognize any elements when tested with the anti-Gal/GalNac antibody (Fig. 1B lane 2).

Oral and nasal immunization with AcNPV-LC3 protects hamsters of developing ALA. Hamsters randomly divided into 6 groups were immunized by mucosal delivery (oral and nasal) with AcNPV-WT, AcNPV-LC3 or $\mathrm{NaHCO}_{3} / \mathrm{PBS}$, before intraportally challenged with virulent trophozoites. Animals were sacrificed 7 days after infection, as illustrated in Fig. 1C.

In order to evaluate the level of protection in relation to development of ALA, we documented a comparative macroscopic description of livers from all experimental groups (massive, partial and absence of abscesses). Thus, 11 out of 19 O AcNPV-LC3 and 4 out of $19 \mathrm{~N}$ AcNPV-LC3 immunized hamsters did not show any macroscopic evidence of ALA development, suggesting sterile protection in these animals (Table 1). In contrast, numerous ALA covering most of the liver's four lobes tissue were clearly evident in all non-immunized sham animals (19 out of 19) as well as in all animals (14 out of 14) immunized with the WT baculovirus (not carrying the LC3 gene) by nasal route and almost all animals (17 out of 19) immunized with WT baculovirus by oral route (Table 1). Same level of ALA development was observed in the other $15 \mathrm{~N}$ AcNPV-LC3 and 4 out of 8 O AcNPV-LC3 non-protected hamsters. In the other four $\mathrm{O}$ AcNPV-LC3 immunized animals, differences in ALA development were observed when comparing sham and WT controls. In general, abscesses were more localized (mostly in one lobe) and smaller, suggesting partial protection. Percentages of protected, partially protected and non-protected animals are shown in Fig. 2A. These differences in ALA development were also observed when comparing the liver weight of all experimental groups, because ALA development is directly related to hepatomegaly. On average, a liver weight of 6 grams was observed in O AcNPV-LC3 
immunized animals, whereas in animals immunized with N AcNPV-LC3 an average of 9 grams was observed. These values were significantly smaller when compared to the sham group (15 g, $p<0.001$ and $p<0.005$; Fig. 2B). Moreover, average liver weight of the O AcNPV-LC3 group was also different from the O AcNPV-WT group (mean of 12 gr; $p<0.005$ ) but not different from the uninfected non-treated group (5gr) (Fig. 2 B). Also, no statistical differences in liver weight between the N AcNPV-LC3 group and its respective WT group were observed.

Macroscopical observations were confirmed through liver section analysis. Thus, no tissue damage or trophozoites were observed in sections from the liver of the 11 orally and 2 nasally AcNPV-LC3 immunized hamsters as well as the 2 orally AcNPV-WT immunized animals with sterile protection (Fig. 3, inset $\mathrm{A}$ and $\mathrm{B})$. In contrast, extensive necrosis in all lobes with tissue liquefaction and scattered trophozoites were observed in all sham and $\mathrm{N}$ AcNPV-WT animals as well as in all N AcNPV-LC3 and O Ac-

A

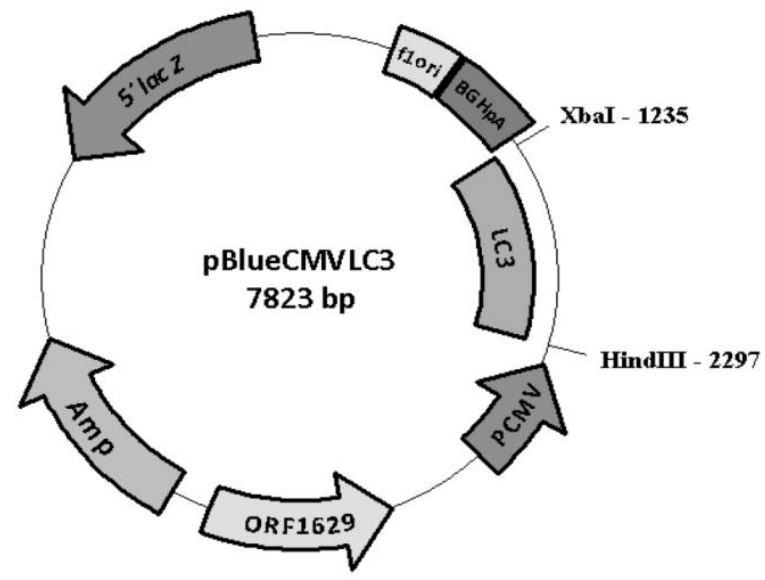

NPV-WT unprotected hamsters (Fig. 3, Insets E to H). The four O AcNPV-LC3 partially protected hamsters showed a considerable reduction in the number and spreading of abscesses ( $\leq 20 \%$ of liver parenchyma) as well as in the number of trophozoites (Fig. 3, inset C and D).

Considering that orally administered WT AcNPV conferred protection at $10.5 \%$, the protection efficacy of AcNPV-LC3 administed by this route was calculated in $57.9 \%$, a value statistically significant regarding sham and O AcNPV-LC3 WT groups $(p<0.05$ by Fisher's test; Table 1$)$. Instead, partial protection was observed in $21 \%$ of the animals (4 out of 19 hamsters) from the O AcNPV-LC3 group, indicating that at least $68.4 \%$ of the animals immunized by this route developed some protection. In contrast, nasally administered AcNPV-LC3 only conferred sterile protection in $21 \%$ of challenged hamsters, with no partial protection observed (Table 1 and Fig. 2A).

\section{B}

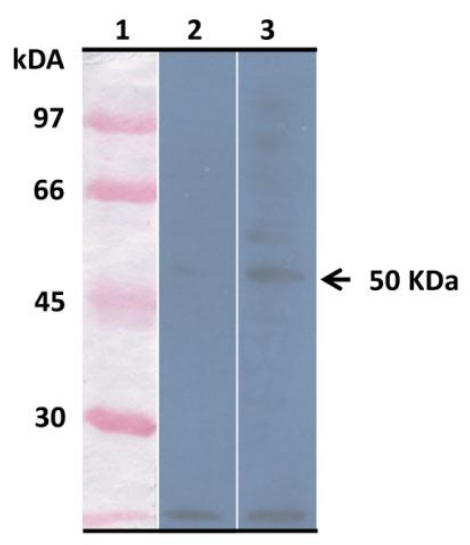

C

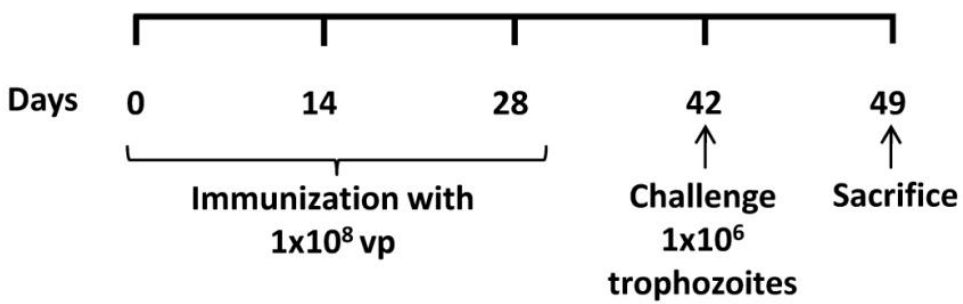

Figure I. Functional characterization of the recombinant ACNPV-LC3 baculovirus and schedule of immunization. AcNPV-LC3 baculovirus was generated by cloning the sequence of the E. histolytica Gal-lectin LC3 fragment into the PBlueCMV transfer plasmid downstream of the CMV promoter sequence (A). Ability of AcNPV-LC3 to drive the expression of LC3 in mammalian cells was carried out by Western blot on transduced HepG2 cells extract using a rabbit polyclonal anti-Gal-lectin heavy subunit antiserum. A band of the expected LC3 molecular weight of $50 \mathrm{kDa}$ was observed in the transduced cells (B, line 3 ). No band was observed in non-transduced cells extract (B, line 2$)$. Details on schedule immunization (oral and nasal routes), intraportal challenge with amoeba and sacrifice of hamsters is shown in C. 
A

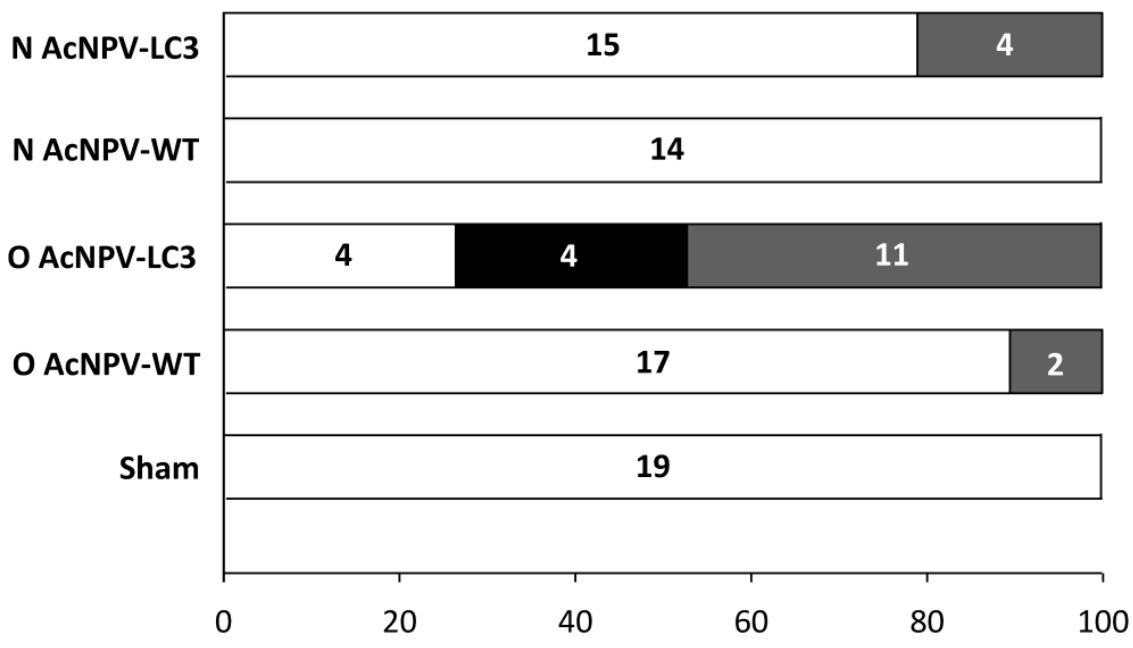

Percentage of protection

B

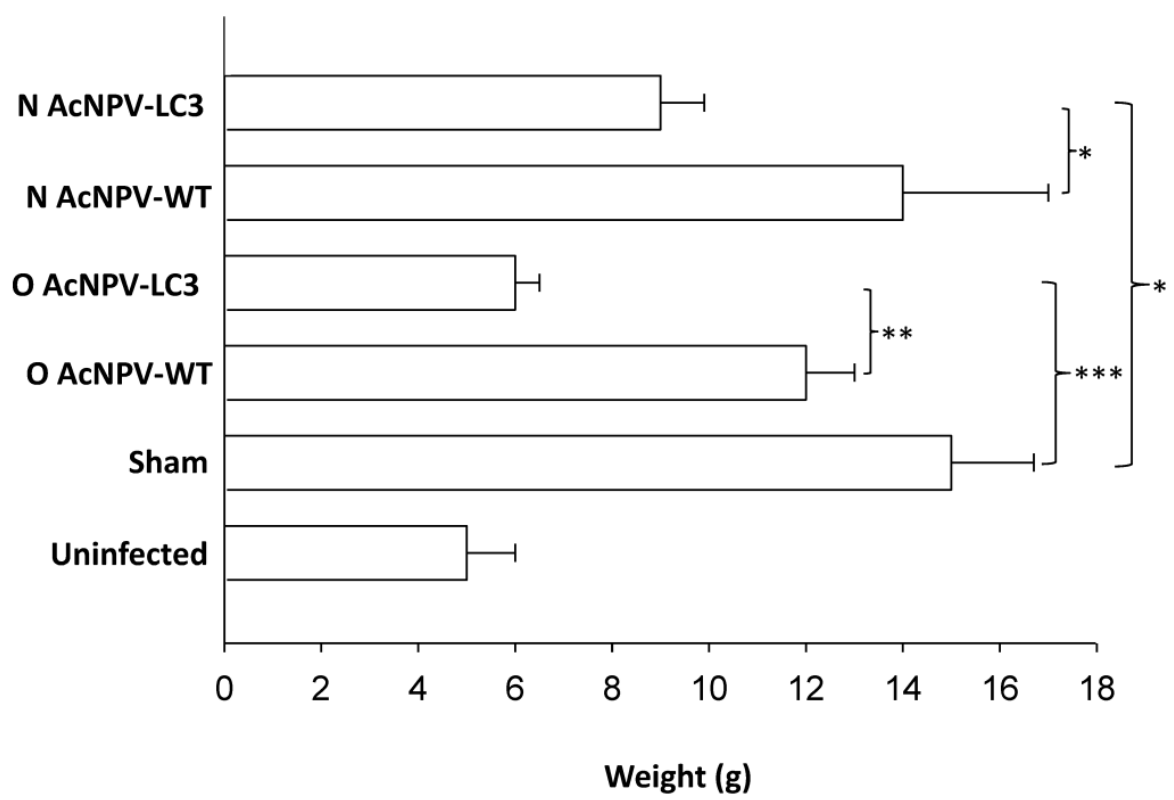

Figure 2. Macroscopical evaluation of protection from ALA challenge in hamsters immunized with AcNPV-LC3. After sacrifice, hamster's livers were exscinded and analyzed for the presence of ALA and hepatomegaly. Percentage and number of infected (open bars), partially protected (ALA in $<20 \%$ of the whole tissue; black filled bars) and sterilizing protection (no evidence of ALA; grey filled bars) for each experimental group is shown in A. Sterilizing protection was observed in AcNPV-LC3 immunized animals, mainly for oral $(\mathrm{O})$ in comparison with nasal $(N)$ routes. Slight sterilizing protection was observed in hamsters receiving WT baculovirus for oral route (O AcNPV-WT). Partial protection was only observed in O AcNPV-LC3. Hepatomegaly recorded as the whole liver weight is shown in B. Bar for each group represents the mean \pm SD (standard deviation) of liver's weigh of all animals assayed. Statistical differences between the oral and nasal AcNPV-LC3 immunized hamsters and the WT and sham control animals are shown $(* p<0.05 ; * * p<0.005 ; * * * p<0.001)$. 


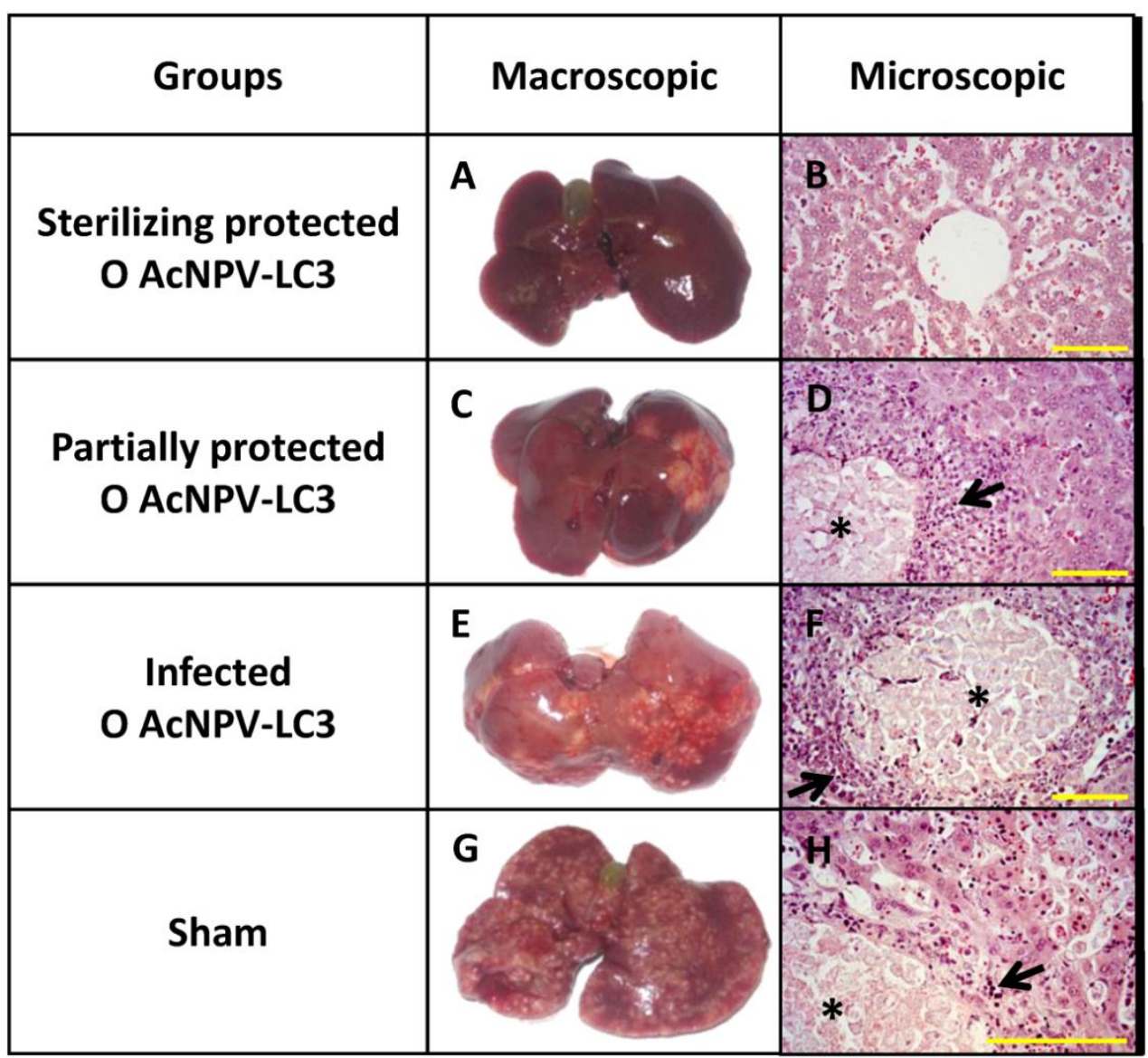

Figure 3. ALA development and hepatic damage analysis in ACNPV-LC3 immunized hamsters after challenge with $E$. histolytica. Representative pictures of the three levels of ALA development observed in hamsters of the $O$ AcNPV-LC3 group, completely protected (sterilizing protection, A), partially protected $(C)$ and non-protected $(E)$. For comparative purposes, one representative liver from an animal of the sham group is shown in G. Hematoxylin-eosin stained section from each liver is shown in $B, D, F$ and $H$, respectively. Healthy liver tissue with no trophozoites was observed in the completely protected hamsters (A and B), in contrast with local (partially protected; $C$ and $D$ ) and spread (unprotected; $E$ to $\mathrm{H}$ ) abscesses, where necrotic focus with multiple trophozoites $\left(^{*}\right)$ surrounded by inflammatory infiltrates (arrow), are usually observed. Yellow bars: $100 \mu \mathrm{m}$.

Table 1. Infection rate in AcNPV-LC3 immunized and untreated control hamsters intraportally infected with E. histolytica trophozoites.

\begin{tabular}{llll}
\hline Groups & $\begin{array}{l}\text { Infection Rate (Infected/Total) } \\
\%\end{array}$ & $p$-value (Fisher's Test) & Protection Efficacy (\%) \\
\hline Sham & $19 / 19$ & NA & 0 \\
O AcNPV-WT & $100 \%$ & & 10.5 \\
& $17 / 19$ & 0.005 & \\
O AcNPV-LC3 & $89.5 \%$ & $($ vs O AcNPV-LC3) & 57.9 \\
& $8 / 19$ & 0.0001 & \\
N AcNPV-WT & $42.1 \%$ & (vs Sham) & 0 \\
& $14 / 14$ & 0.1192 & \\
N AcNPV-LC3 & $100 \%$ & (vs N AcNPV-LC3) & 21 \\
& $15 / 19$ & 0.1 & \\
& $79 \%$ & (vs Sham) & \\
\hline
\end{tabular}


Oral and nasal vaccination with AcNPV-LC3 induces high levels of systemic anti-amoeba antibodies. Systemic antibodies raised against LC3 by immunization with the recombinant baculovirus were determined by ELISA in pre and post-immune sera collected from all hamsters. High titer of anti-trophozoite antigen IgG antibodies was observed in serum from $\mathrm{O}$ AcNPV-LC3 and $\mathrm{N}$ AcNPV-LC3 groups after immunization; in contrast to the respective oral and nasal WT immunized groups which did not developed $\operatorname{IgG}$ antibodies recognizing amoeba extracts $(p<0.001$ for oral groups and $p<0.005$ for the nasal groups comparisons; Fig. 4A, open bars).

A

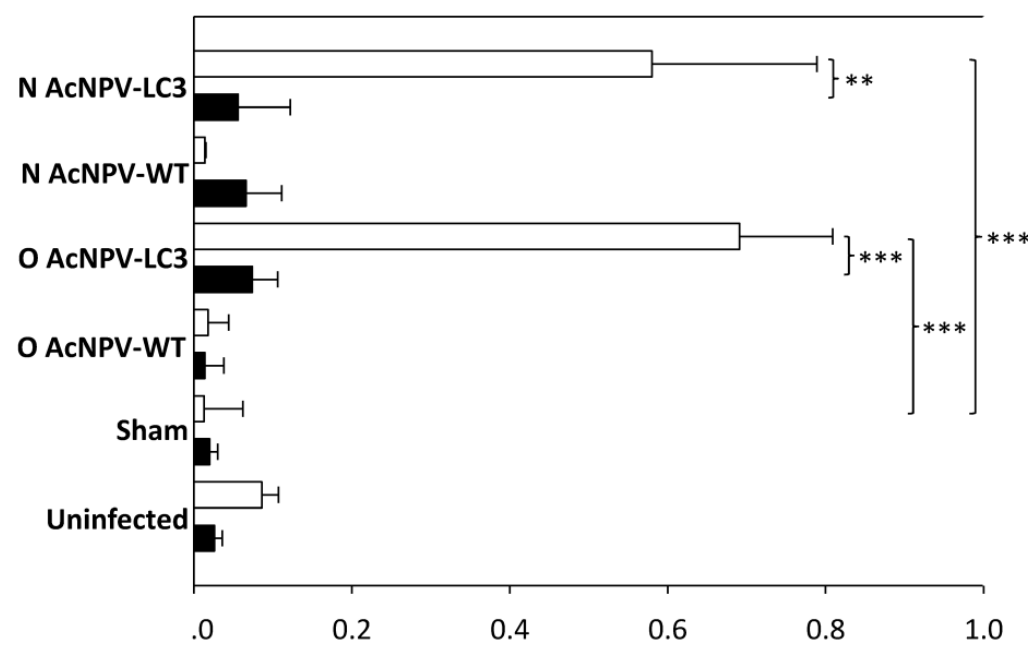

B

Absorbance $490 \mathrm{~nm}$

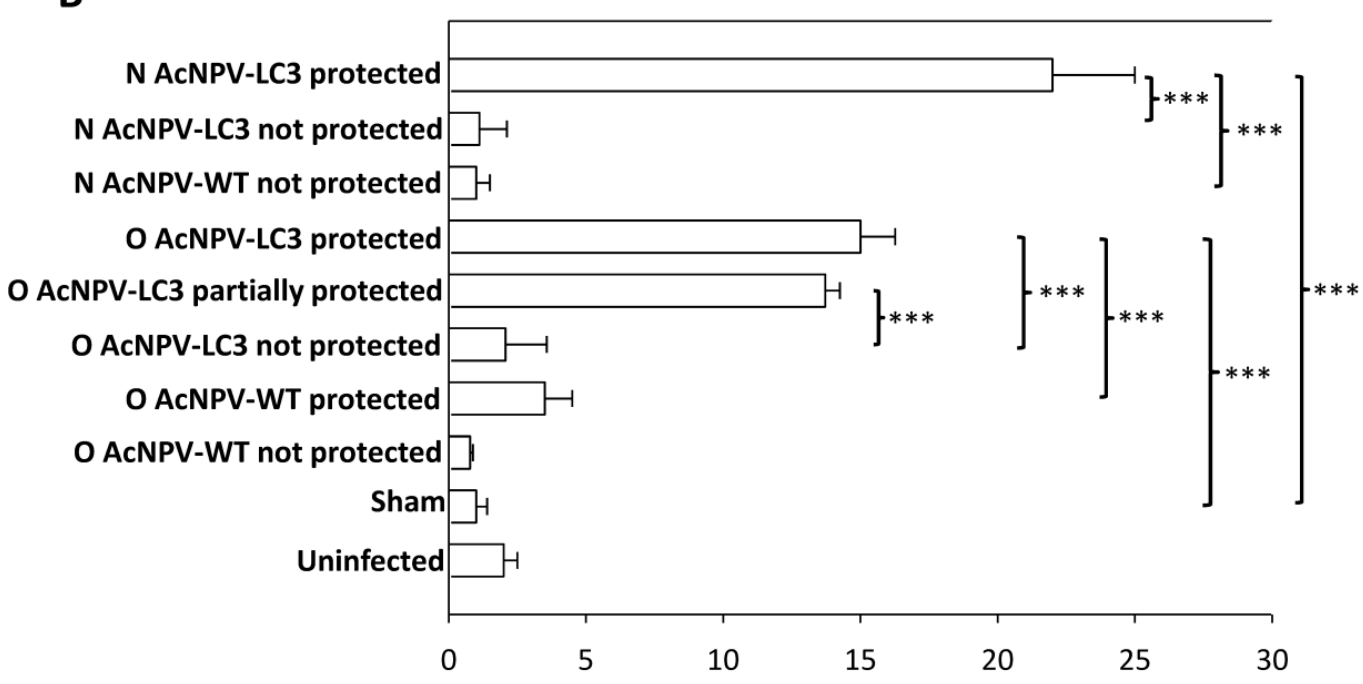

Proliferation rate

Figure 4. Humoral and cellular anti-E. histolytica immune responses in ACNPV-LC3 immunized and challenged hamsters. A. IgG antibodies in sera against total extract of $E$. histolytica trophozoites were measured by ELISA at day 0 (pre-immune sera, filled bars) and 7 days prior to intraportal challenge with trophozoites (day 35; post-immune sera, open bars). Bars represent mean optical densities $(n=19)$. High level of specific $\lg G$ antibodies were detected only in sera from LC3 immunized animals, independent of the route of immunization $(* * p<0.005 ; * * * p<0.00 \mathrm{I})$. B. Cellular immune response was evaluated in splenocytes at the day of sacrifice (day 49) by staining of the harvested cells with CFSE and stimulation with E. histolytica total extract during $72 \mathrm{~h}$. Bars represent mean of percentage of proliferation of the number of hamsters include in each classification. Specific proliferation was only observed in the protected animals, independent of the route of immunization (**p $<0.005$; *** $p<0.001)$. 
Sera from the AcNPV-LC3 immunized hamsters by both routes recognized a band around $170 \mathrm{kDa}$ in amoebic total extract by Western blot, suggesting that antibodies specific against LC3 and therefore, for the Gal-lectin heavy subunit were induced in the animals (data not shown). No systemic response was observed in the sham and uninfected control groups $(p<0.001$ when comparing with AcNPV-LC3 immunized groups). These result of high level of IgG antibodies in hamsters immunized by both routes with no clear differences between them, suggest that anti-LC3 IgG antibodies may be only partially responsible for protection against ALA, as clear differences in the level of protection between the oral and nasal AcNPV-LC3 immunized groups were observed (Table 1 and Fig. 2A).

\section{Oral and nasal vaccination with AcNPV-LC3 induces cell-mediated immune responses.} Immunization-based lymphocyte priming was determined in all animals by assaying spleen cells proliferation upon stimulation with E. histolytica trophozoite's extracts. As shown in Fig. 4B, all protected animals (sterile and partial) by immunization with AcNPV-LC3 showed high proportion of proliferating cells upon exposition to trophozoite's antigens, independent of the route of immunization. Mean proliferation values of $15 \%$ and $22 \%$ of the total population for the oral and nasal route respectively were observed in the animals with sterile protection. Mean proliferation of $13 \%$ of the total population was also observed for the partially protected animals by oral route. In contrast, only cellular background proliferation was observed in the unprotected AcNPV-LC3 and WT immunized hamsters, including the two $\mathrm{O}$ AcNPV-WT protected hamsters $(p<0.005$ comparing protected O AcNPV-LC3 versus unprotected O AcNPV-LC3 and O-AcNPV-WT groups; $p<0.001$ comparing protected $\mathrm{N}$ AcNPV-LC3 vs unprotected $\mathrm{N}$ AcNPV-LC3 and N-AcNPV-WT groups). Similar background proliferation was also found for sham and uninfected control groups (Fig. 4B). These results suggest that protection against ALA in hamsters by mucosal immunization with baculovirus driving the expression of LC3, was partially dependent on antibodies but strongly dependent on the induction of a specific cellular immune response in spleen.

\section{Discussion}

E. histolytica Gal-lectin has been shown to play an important role in parasite colonization, cytotoxicity, complement resistance and encystment. Capable of activating dendritic cells and macrophages in vitro, the Gal-lectin is also an immunodominant antigen recognized by sera from patients carrying amoebic infections $(16,44)$. The combination of these factors poses E. histolytica Gal-lectin as the leading vaccine candidate against human amoebiasis (45). In the present work, we show for the first time that oral and nasal immunization of hamsters with the baculovirus A. californica driving the expression of the immunogenic E. histolytica Gal-lectin heavy chain LC3 fragment resulted in the induction of systemic humoral and cellular anti-LC3 immune responses that conferred protection against ALA. Protection was observed at two levels: i) sterilizing protection, with no evidence of macroscopic or microscopic lesions and no liver trophozoites, observed in $57.9 \%$ and $21 \%$ of hamster groups immunized with O AcNPV-LC3 or N AcNPV-LC3, respectively (Table 1); and ii) partial protection, with reduction in ALA sizes and weights, observed in the O AcNPV-LC3 immunized group (21\%; Fig. 2A and Table 1). Sterilizing protection observed in the O AcNPV-LC3 group suggests that the adaptive immune response elicited upon immunization, measured as anti-amoeba IgG antibodies in sera and spleen cells proliferation, was sufficient to avoid early colonization of liver tissue by trophozoites upon challenge. These results are evidence of immune recall and thereof immunological memory (46). However, complete protection against ALA by both routes (17 out of 19 hamsters by oral and 4 out of 19 by nasal) was only correlated with the lymphoproliferation measured from spleens (Fig. 4B) but not with the level of anti-amoeba IgG antibodies, as no differences in the level of such antibodies were found when comparing protected vs unprotected AcNPV-LC3 immunized hamsters (Fig. 4A).

Our protection results agree with those reported by Ivory and Chadee where gerbils nasally immunized with the Gal-lectin heavy chain and CpG oligonucleotides as TLR-9 adjuvant were protected from ALA demonstrating, as we report here, that an effective immune response was raised after mucosal vaccination. However, in contrast to this report, we reached only $21 \%$ of sterile protection by nasal immunization whereas they observed partial protection before 5 days and almost sterilizing protection at 10 and 15 days (10). Moreover, in counterpart to our result of AcNPV adjuvant activity conferring slight protection by itself, they observed that CpG-treated gerbils developed larger abscesses than those excised from PBS-treated animals, suggesting that this exacerbation could be due to the ability of CpG-ODN to activate innate immune cells through TLR9, a mechanism that might be shared with AcNPV (10). Although the mechanisms underlying these differences are unknown, the different protection levels could be due to differences in the animal model, 
trophozoites virulence and adjuvants used for each study, as well as to differences in the compartmentalization of the induced immune responses. In control groups, low sterilizing protection $(10.5 \%)$ was only observed in O AcNPV-WT, which showed neither correlation with spleenocytes response to amoebic extract (Fig. 4B) nor cross-reactive antibodies to amoeba (Fig. 4A). The reason for the sterilizing protection observed in these two animals is unknown, but may be related to poor infection rates during the challenge with trophozoites. Protection due to baculovirus activation of innate immunity is unlikely, because of the low number of animals protected in the WT control groups. Taking together, our study in combination with those the mentioned above, and others conferring protection against ALA in gerbils by oral immunization with attenuated bacteria such as Yersinia enterocolitica and Salmonella Typhimurium, support the concept that mucosal immunization, and particularly the oral route, as a useful strategy for delivery of an effective anti-amoebic vaccine, not only against intestinal amoebiasis, but also effective against a systemic challenge. In this sense, oral vaccination is considered to be a highly desirable form of vaccination, being noninvasive, painless, and self-administrable (47).

On the other hand, the baculovirus-based delivery system we used here, may offer an attractive immunization strategy because of the low cytotoxicity and inability of the virus to replicate in mammalian cells (27). Baculovirus are known to efficiently drive the expression of foreign proteins in mammalian cells, being currently used for large-scale production of recombinant proteins of clinical utility (48), and recently proposed as a novel tool for vector-based human vaccines (49). Since baculovirus can only drive the expression of LC3 once inside a cell, our results also indicate that AcNPV administered by oral and nasal routes were able to transduce cells with antigen presentation potential, such as local DCs or macrophages in the intestinal submucosa and Peyer's patches, which in turn processed the LC3 fragment and present it to $\mathrm{T}$ and $\mathrm{B}$ lymphocytes in the draining mesenteric nodes, resulting in the specific systemic responses and varying degrees of protection observed in immunized animals. However, studies of AcNPV cell targeting in immunized hamsters by using labeled baculovirus should be carried out in order to demonstrate in vivo transduction of APC and other immune cells by the baculovirus. Our results are in agreement with many in vivo and in vitro studies showing baculovirus as potent adjuvants by itself, inducing DCs maturation by TLR9 recognition through MyD88-dependent and independent path- ways (28), and increasing its capacity of activate $\mathrm{T}$ cells towards a Th1-biased inflammatory response (50). Previous studies of protection against intestinal and hepatic amoebiasis have used CT as adjuvant (51-53), a strong immune elicitor that can induce immunological memory against heterologous antigens in most animal models. However, CT is no authorized for clinical use in humans due to its enterotoxicity and potential harmful effects on olfactory nerves when administered nasally (54).

Mechanisms underlying hamster protection against ALA conferred by the oral administration of AcNPV-LC3 remain largely unknown, but correlations with anti-amoeba cellular responses measured in the spleen were observed (Fig. 4 A and B). Phenotypic and functional maturation of dendritic cells and macrophages as well as direct activation of B cells induced by AcNPV promotes antigen presentation and induction of a Th1 response and IgG2a antibodies, as shown in numerous studies $(28,29,50)$. The AcNPV adjuvant effect could be indispensable for the sterilizing protection observed in the liver, breaking the tolerogenic environment present in physiological conditions because of exposition to dietary antigens and microbe's metabolites that are produced locally, as also occurs in the intestine (55). Thus, it has been suggested that during an acute $E$. histolytica infection in the liver parenchyma, the functional activation of iNKT, macrophages and Th1 cells is needed to both, control infection and downregulate the effect of resident cells such as DCs, Kupffer cells and Tregs, whose activation usually induces tolerance (56). Such mechanism could be present also in the intestine, where oral immunization with AcNPV-LC3 could induce local IgA and cellular immune responses, in addition to the systemic response reported here. One can speculate that a similar mechanism could prevent large intestine colonization by E. histolytica, therefore preventing development of human intestinal amoebiasis and ALA consequently.

Further studies using alternative strategies such as protection studies in hamsters and mice passively transferred with lymphoid cells from protected animals may provide sufficient information to identify the mechanisms underlying liver and intestine sterilizing protection by oral immunization observed in this work.

\section{Acknowledgments}

This work was supported by CONACyT Grants 61111 (JCC) and 61334 (JPL), and by DGAPA Grants IN-227707 (JCC), IN213611-3 (JCC) and IN-230207 (JPL). Dulce María Meneses Ruiz thanks to CONACyT for a fellowship supporting the PhD studies. She 
also wants to thank to the program "Doctorado en Ciencias Biomédicas" and to the UNAM. We thank Mario Nequiz for amoeba culturing and Pedro Balderas for histology assistant.

\section{Conflict of Interests}

The authors have declared that no conflict of interest exists.

\section{References}

1. [No authors listed]. WHO: Geneva, Switzerland. Weekly Epi-demiological Record. Amoebiasis from 4 April 1997; 72: 97-100.

2. Ximénez C, Morán P, Rojas L, et al. Reassessment of the epi-demiology of amebiasis: state of the art. Infect Genet Evol. 2009; 9: 1023-32.

3. Caballero-Salcedo A, Viveros-Rogel M, Salvatierra B, et al. Seroepidemiology of amebiasis in Mexico. Am J Trop Med Hyg. 1994; 50: 412-9.

4. Gaucher D, Chadee K. Prospect for an Entamoeba histolytica Gal-lectin-based vaccine. Parasite Immunol. 2003; 25: 55-8.

5. Haque R, Kabir M, Noor Z, et al. Diagnosis of amebic liver abscess and amebic colitis by detection of Entamoeba histolytica DNA in blood, urine, and saliva by a Real-Time PCR assay. J Clin Microbiol. 2010; 48: 2798-801.

6. Lotter $\mathrm{H}$, Tannich $\mathrm{E}$. The current status of an amebiasis vaccine. Arch Med Res. 2006; 37: 292-6.

7. Zhang T, Cieslak PR, Stanley SL Jr. Protection of gerbils from amebic liver abscess by immunization with a recombinant En-tamoeba histolytica antigen. Infect Immun. 1994; 62: 1166-70.

8. Soong CJ, Kain KC, Abd-Alla M, et al. A recombinant cyste-ine-rich section of the Entamoeba histolytica galac-tose-inhibitable lectin is efficacious as a subunit vaccine in the gerbil model of amebic liver abscess. J Infect Dis. 1995; 171: 645-51.

9. Ivory $\mathrm{CP}$, Keller K, Chadee K. CpG-oligodeoxynucleotide is a potent adjuvant with an Entamoeba histolytica Gal-inhibitable lectin vaccine against amoebic liver abscess in gerbils. Infect Immun. 2006; 74: 528-36.

10. Ivory $\mathrm{CP}$, Chadee $\mathrm{K}$. Intranasal immunization with Gal-inhibitable lectin plus an adjuvant of CpG oligodeoxynu-cleotides protects against Entamoeba histolytica challenge. Infect Immun. 2007;75: 4917-22.

11. Lotter H, Rüssmann H, Heesemann J, Tannich E. Attenuated recombinant Yersinia as live oral vaccine carrier to protect against amoebiasis. Int J Med Microbiol. 2008; 298: 79-86.

12. Mann BJ, Burkholder BV, Lockhart LA. Protection in a gerbil model of amebiasis by oral immunization with Salmonella ex-pressing the galactose/ $\mathrm{N}$-acetyl D-galactosamine inhibitable lectin of Entamoeba histolytica. Vaccine. 1997; 15: 659-63.

13. Cheng XJ, Tachibana H, Kaneda Y. Protection of hamsters from amebic liver abscess formation by a monoclonal antibody to a 150-kDa surface lectin of Entamoeba histolytica. Parasitol Res. 1999; 85: 78-80.

14. Abd-Alla MD, Jackson TF, Reddy S, et al. Diagnosis of invasive amebiasis by enzyme-linked immunosorbent assay of saliva to detect amebic lectin antigen and anti-lectin immunoglobulin $G$ antibodies. J Clin Microbiol. 2000; 38: 2344-7.

15. Abou-el-Magd I, Soong CJ, el-Hawey AM, et al. Humoral and mucosal IgA antibody response to a recombinant $52-\mathrm{kDa}$ cys-teine-rich portion of the Entamoeba histolytica galac-tose-inhibitable lectin correlates with detection of native
170-kDa lectin antigen in serum of patients with amebic colitis. J Infect Dis. 1996; 174: 157-62.

16. Abd-Alla MD, Jackson TF, Soong GC, et al. Identification of the Entamoeba histolytica galactose-inhibitable lectin epitopes recognized by human immunoglobulin A antibodies following cure of amebic liver abscess. Infect Immun. 2004; 72: 3974-3980.

17. Beving DE, Soong CJ, Ravdin JI. Oral immunization with a recombinant cysteine-rich section of the Entamoeba histolytica galactose-inhibitable lectin elicits an intestinal secretory im-munoglobulin A response that has in vitro adherence inhibition activity. Infect Immun. 1996; 64: 1473-6.

18. Abd Alla MD, White GL, Rogers TB, et al. Adherence-inhibitory intestinal immunoglobulin a antibody response in baboons elicited by use of a synthetic intranasal lectin-based amebiasis subunit vaccine. Infect Immun. 2007; 75: 3812-22.

19. Zhang T, Stanley SL Jr. Protection of gerbils from amebic liver abscess by immunization with a recombinant protein derived from the 170-kilodalton surface adhesin of Entamoeba histo-lytica. Infect Immun. 1994; 62: 2605-8.

20. Zhang T, Stanley SL Jr. Oral immunization with an attenuated vaccine strain of Salmonella typhimurium expressing the ser-ine-rich Entamoeba histolytica protein induces an antiamebic immune response and protects gerbils from amebic liver ab-scess. Infect Immun. 1996; 64: 1526-31.

21. Lotter H, Khajawa F, Stanley SL Jr, et al. Protection of gerbils from amebic liver abscess by vaccination with a 25 -mer peptide derived from the cysteine-rich region of Entamoeba histolytica galactose-specific adherence lectin. Infect Immun. 2000; 68: 4416-21.

22. Cheng XJ, Tachibana H. Protection of hamsters from amebic liver abscess formation by immunization with the 150- and 170-kDa surface antigens of Entamoeba histolytica. Parasitol Res. 2001; 87: 126-30.

23. Gaucher D, Chadee K. Construction and immunogenicity of a codon-optimized Entamoeba histolytica Gal-lectin-based DNA vaccine. Vaccine. 2002; 20: 3244-3253.

24. Lotter H, Rüssmann H, Heesemann J, Tannich E. Oral vaccina-tion with recombinant Yersinia enterocolitica expressing hybrid type III proteins protects gerbils from amebic liver abscess. Infect Immun. 2004; 72: 7318-21.

25. Jiménez-Delgadillo B, Chaudhuri PP, Baylón-Pacheco L, et al. Entamoeba histolytica: cDNAs cloned as $30 \mathrm{kDa}$ colla-gen-binding proteins (CBP) belong to an antioxidant molecule family. Protection of hamsters from amoebic liver abscess by immunization with recombinant CBP. Exp Parasitol. 2004; 108: 7-17.

26. Martínez MB, Rodríguez MA, García-Rivera G, et al. A pcDNA-Ehcpadh vaccine against Entamoeba histolytica elicits a protective Th1-like response in hamster liver. Vaccine. 2009; 27: 4176-86.

27. Shoji I, Aizaki H, Tani H, et al. 1997. Efficient gene transfer into various mammalian cells, including non-hepatic cells, by bac-ulovirus vectors. J Gen Virol. 1997; 78: 2657-2664.

28. Abe $T$, Hemmi $H$, Miyamoto $H$, et al. Involvement of the Toll-Like Receptor 9 signaling pathway in the induction of in-nate immunity by baculovirus. J Virol. 2005; 70: 2847-2858.

29. Hervas-Stubbs, Rueda P, Lopez L, et al. Insect baculoviruses strongly potentiate adaptive immune responses by inducing type I IFN. J Immunol. 2007; 178: 2361-2369.

30. Kaba SA, Hemmes JC, van Lent JW, et al. Baculovirus surface display of Theileria parva p67 antigen preserves the confor-mation of sporozoite-neutralizing epitopes. Protein Eng. 2003; 16: 73-78.

31. Rahman MM, Shaila MS, Gopinathan KP. Baculovirus display of fusion protein of Peste des petits ruminants virus and he-magglutination protein of Rinderpest virus and immuno- 
genic-ity of the displayed proteins in mouse model. Virology. 2003; 317: 36-49.

32. Yoshida S, Kondoh D, Arai E, et al. Baculovirus virions dis-playing Plasmodium berghei circumsporozoite protein protect mice against malaria sporozoite infection. Virology. 2003; 316: 161-70.

33. Feng Q, Liu Y, Qu X, et al. Baculovirus surface display of SARS Coronavirus (SARS-CoV) spike protein and immunogenicity of the displayed protein in mice models. DNA Cell Biol. 2006; 25: 668-673.

34. Strauss R, Hüser A, Ni S, et al. Baculovirus-based vaccination vector a low for efficient induction of immune responses against Plasmodium falciparum circumsporozoite protein. Mol Ther. 2007; 15: 193-202.

35. Peralta A, Molinari P, Conte-Grand D, et al. A chimeric bacu-lovirus displaying bovine herpesvirus-1 (BHV-1) glycoprotein $\mathrm{D}$ on its surface and their immunological properties. Appl Mi-crobiol Biotechnol 2007; 75: 407-414.

36. Prabakaran M, Velumani S, He F, et al. Protective immunity against influenza $\mathrm{H} 5 \mathrm{~N} 1$ virus challenge in mice by intranasal co-administration of baculovirus surface-displayed HA and recombinant CTB as an adjuvant. Virology. 2008; 380: 412-20.

37. Yoshida S, Kawasaki M, Hariguchi N, et al. A baculovirus dual expression system-based malaria vaccine induces strong pro-tection against Plasmodium berghei sporozoite challenge in mice. Infect Immun. 2009; 77: 1782-9.

38. Wu Q, Fang L, Wu X, et al. A pseudotype baculovirus-mediated vaccine confers protective immunity against lethal challenge with H5N1 avian influenza virus in mice and chickens. Mol Immunol. 2009; 46: 2210-7.

39. Yoshida S, Araki H, Yokomine T. Baculovirus-based nasal drop vaccine confers complete protection against malaria by natural boosting of vaccine-induced antibodies in mice. Infect Immun. 2010; 78: 595-602.

40. Prabakaran M, Madhan S, Prabhu N, et al. Gastrointestinal delivery of baculovirus displaying influenza virus hemaggluti-nin protects mice against heterologous H5N1 infection. J Virol. 2010; 84: 3201-9.

41. Blagborough AM, Yoshida S, Sattabongkot J, et al. Intranasal and intramuscular immunization with Baculovirus Dual Ex-pression System-based Pvs25 vaccine substantially blocks Plasmodium vivax transmission. Vaccine. 2010; 28: 6014-20.

42. Luz-Madrigal A, Clapp C, Aranda J, et al. In vivo transcriptional targeting into the retinal vasculature using recombinant baculovirus carrying the human flt-1 promoter. Virol J. 2007;4:88.

43. Olivos-García A, Carrero JC, Ramos E, et al. Late experimental amebic liver abscess in hamster is inhibited by cyclosporine and N-acetylcysteine. Exp Mol Pathol. 2007; 82: 310-5.

44. Séguin R, Mann BJ, Keller K, et al. Identification of the galac-tose-adherence lectin epitopes of Entamoeba histolytica that stimulate tumor necrosis factor-alpha production by macro-phages. Proc Natl Acad Sci. 1995; 92: 12175-79.

45. Petri WA Jr, Broman J, Healy G, et al. Antigenic stability and immunodominance of the Gal/GalNAc adherence lectin of Entamoeba histolytica. Am J Med Sci. 1989; 297: 163-165.

46. Schain DC, Salata RA, Ravdin JI. Development of amebicidal cell-mediated immunity in gerbils (Meriones unguiculatus) immunized with the galactose-inhibitable adherence lectin of Entamoeba histolytica. J Parasitol. 1995; 81: 563-8.

47. Azizi A, Kumar A, Diaz-Mitoma F, et al. Enhancing oral vaccine potency by targeting intestinal M cells. PLoS Pathog. 2010; 6: e1001147.

48. Kost TA, Condreay JP, Jarvis DL. Baculovirus as versatile vectors for protein expression in insect and mammalian cells. Nat Biotechnol. 2005; 23: 567-75.
49. Madhan S, Prabakaran M, Kwang J. Baculovirus as vaccine vectors. Curr Gene Ther. 2010; 10: 201-13.

50. Schütz A, Scheller N, Breinig T, et al. The Autographa califor-nica nuclear polyhedrosis virus AcNPV induces functional maturation of human monocyte derived dendritic cells. Vaccine 2006; 24: 7190-6.

51. Carrero JC, Contreras-Rojas A, Sánchez-Hernández B, et al. Protection against murine intestinal amoebiasis induced by oral immunization with the $29 \mathrm{kDa}$ antigen of Entamoeba histolytica and cholera toxin. Exp Parasitol. 2010; 126: 359-65.

52. Guo X, Barroso L, Becker SM, et al. Protection against intestinal amebiasis by a recombinant vaccine is transferable by $\mathrm{T}$ cells and mediated by gamma interferon. Infect Immun. 2009; 77: 3909-18.

53. Houpt E, Barroso L, Lockhart L, et al. Prevention of intestinal amebiasis by vaccination with the Entamoeba histolytica Gal/GalNac lectin. Vaccine. 2004; 22: 611-7.

54. Hagiwara $\mathrm{Y}$, Iwasaki $\mathrm{T}$, Asanuma $\mathrm{H}$, et al. Effects of intranasal administration of cholera toxin (or Escherichia coli heat-labile enterotoxin) B subunits supplemented with a trace amount of the holotoxin on the brain. Vaccine. 2001; 19: 1652-60.

55. Thomson AW, Knolle PA. Antigen-presenting cell function in the tolerogenic liver environment. Nat Rev Immunol. 2010; 10: 753-66.

56. Mortimer L, Chadee K. The immunopathogenesis of Entamoeba histolytica. Exp Parasitol. 2010; 126: 366-80. 